\title{
RINGS AND SUBRINGS OF CONTINUOUS FUNCTIONS WITH COUNTABLE RANGE
}

\author{
SUDIP KUMAR ACHARYYA, RAKESH BHARATI, AND A. DEB RAY
}

\begin{abstract}
Intermediate rings of real valued continuous functions with countable range on a Hausdorff zero-dimensional space $X$ are introduced in this article. Let $\Sigma_{c}(X)$ be the family of all such intermediate rings $A_{c}(X)$ 's which lie between $C_{c}^{*}(X)$ and $C_{c}(X)$. It is shown that the structure space of each $A_{c}(X)$ is $\beta_{0} X$, the Banaschewski compactification of $X . X$ is shown to be a $P$-space if and only if each ideal in $C_{c}(X)$ is closed in the $m_{c}$-topology on it. Furthermore $X$ is realized to be an almost $P$-space when and only when each maximal ideal $/ z$-ideal in $C_{c}(X)$ becomes a $z^{0}$-ideal. Incidentally within the family of almost $P$-spaces, $C_{c}(X)$ is characterized among all the members of $\Sigma_{c}(X)$ by virtue of either of these two properties. Equivalent descriptions of pseudocompact condition on $X$ are given via $U_{c}$-topology, $m_{c}$-topology and norm on $C_{c}(X)$. The article ends with a result which essentially says that $z^{0}$-ideals in a typical $A_{c}(X) \in \Sigma_{c}(X)$ are precisely the contraction of $z^{0}$-ideals in $C_{c}(X)$.
\end{abstract}

\section{INTRODUCTION}

In what follows $X$ stands for a completely regular Hausdorff topological space and $C(X)$ as usual denotes the ring of all real valued continuous functions on $X$. $C^{*}(X)$ designates the subring of $C(X)$ containing all those members which are bounded over $X$. Suppose $C_{c}(X)$ is the subset of $C(X)$ consisting of those functions $f$ for which $f(X)$ is a countable subset of $\mathbb{R}$ and $C_{c}^{*}(X)=C_{c}(X) \cap C^{*}(X)$. It is well known that $C_{c}(X)$ (respectively $C_{c}^{*}(X)$ ) is a subring as well as a sublattice of $C(X)$ (respectively $C^{*}(X)$ ). These two rings $C_{c}(X)$ and $C_{c}^{*}(X)$ have received the attention of a few experts in this area only recently. We refer to the reader the articles [5, [11, 21], [16] in this connection. A natural expectation has cropped up as a bye-product of these recent investigations that, there is a hidden interplay existing between the topological structure of $X$ and the ring and the lattice structure of $C_{c}(X)$ and $C_{c}^{*}(X)$. To study this interaction in an efficient manner the authors in [14] have already discovered that one can stick to a well chosen class of spaces viz. the zero-dimensional Hausdorff topological space $X$. Indeed it is proved in ([14], Theorem 4.6) that starting from any topological space $X$ (not necessarily even completely regular), one can construct a Hausdorff zero-dimensional space $Y$ such that the ring $C_{c}(Y)$ is isomorphic to the ring $C_{c}(X)$. This may be called the analogous fact for its classical antecedent in the theory of $C(X)$ which says that any topological space $X$ can give rise to a completely regular Hausdorff space $Y$

2010 Mathematics Subject Classification. 54C40.

$K e y$ words and phrases. Intermediate rings, zero-dimensional space, $P$-spaces, almost $P$ spaces, $m_{c}$-topology, $z^{0}$-ideals, Banaschewski compactification.

The second author acknowledges financial support from University Grand Commission, New Delhi, for the award of research fellowship (File No. 16-9(June 2018)/2019 (NET/CSIR)). 
for which $C(X)$ is isomorphic to $C(Y)$ (13], Theorem 3.9). Therefore in the study of $C_{c}(X)$ and $C_{c}^{*}(X)$ vis-a-vis the space $X$ the ambient topological space $X$ may well chosen to be Hausdorff and zero-dimensional in the sense that clopen sets make base for the topology on $X$. We will stick to this convention throughout this article. Furthermore an ideal $I$ unmodified in any ring $\mathrm{R}$ in this paper will always stand for a proper ideal.

It is a standard result in the theory of Rings of Continuous functions that the

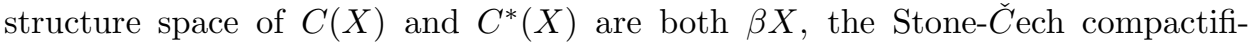
cation of $X(7 \mathrm{~N},[13)$. As a countable counterpart of the result, it is proved in ( 5 , Remark 3.6) that the structure space of $C_{c}(X)$ is $\beta_{0} X$, the largest zerodimensional compactification of a zero-dimensional Hausdorff space $X$, also known as Banaschewski compactification of $X$. The structure space of a commutative ring $R$ with unity stands for the set of all maximal ideals of $R$ equipped with the familiar hull-kernel topology. In the present article we have initiated the study on intermediate rings viz those rings that lie between $C_{c}^{*}(X)$ and $C_{c}(X)$. Let $\Sigma_{c}(X)$ stand for the aggregate of all such intermediate rings. In section 2 of the present article we establish that if $A_{c}(X) \in \Sigma_{c}(X)$ then the structure space of $A_{c}(X)$ is also $\beta_{0} X$ (Theorem 2.7). This generalizes the Proposition mentioned in 5. This is incidentally the first important technical result in this article. A space $\mathrm{X}$ is termed as a $C P$-space in 14 if the ring $C_{c}(X)$ is regular in the sense of Von-Neumann and several equivalent versions of this property are recorded in ([14, Theorem 5.8). These are natural counterparts of the corresponding equivalent descriptions of a $P$-space in the classical setting of $C(X)$ as mentioned in (4J, [13]). It is also proved in the same article ([14, Corollary 5.7) that a zero-dimensional space $X$ is a $C P$ space if and only if it is a $P$-space. In section 3 of the present article we introduce $m_{c}$-topology on $C_{c}(X)$ as a counterpart for the present set up of the well known $m$ topology on $C(X)$, introduced longtime back by Hewitt in 1948 [15]. We prove that if $I$ is an ideal of $C_{c}(X)$, then the closure of $I$ in the $m_{c}$-topology coincides with the intersection of all the maximal ideals of $C_{c}(X)$ which contain $I$ (Theorem 3.8). From this it follows that a zero-dimensional space $X$ is $P$-space if and only if each ideal in $C_{c}(X)$ is closed in $m_{c}$-topology (Theorem 3.10). We further establish that if $A_{c}(X) \in \Sigma_{c}(X)$ is properly contained in $C_{c}(X)$ then it is never Von-Neumann regular (Theorem 3.16). Thus within the class of $P$-spaces $X, C_{c}(X)$ is characterized amongst all the intermediate rings by the property that it is Von-Neumann regular.

A Tychonoff space $X$ is called almost $P$ if the interior of each non empty zero set in $X$ is open. These spaces are introduced in [18] as a generalization of $P$-spaces. In section 4 of this article we make some query about when a zero-dimensional space $X$ becomes an almost $P$-space. We establish that $X$ is almost $P$ if and only if each maximal ideal of $C_{c}(X)$ is a $z^{0}$-ideal and this happens when and only when each $z$-ideal in $C_{c}(X)$ becomes a $z^{0}$-ideal (Theorem 4.10). It turns out that within the class of almost $P$-space $X, C_{c}(X)$ is the unique ring amongst all the intermediate rings that lie between $C_{c}^{*}(X)$ and $C_{c}(X)$ which enjoys either of these two properties (Theorem 4.114.12).

A space $X$ is called pseudocompact if $C(X)=C^{*}(X)$. It is established by the authors in (16], Theorem 6.3) that a zero-dimensional space $X$ is pseudocompact if and only if $C_{c}(X)=C_{c}^{*}(X)$. In section 5 of this article we find out a few equivalent 
versions of pseudocompactness in terms of both the $m_{c^{-}}$topology on $C_{c}(X)$ and $U_{c^{-}}$ topology on $C_{c}(X)$ (Theorem 5.2, 5.3). The $U_{c}$-topology on $C_{c}(X)$ may be called the countable counterpart of the well known $U$-topology or the topology of uniform convergence on $C(X)$ (See $2 \mathrm{M}, 2 \mathrm{~N},[13$ ).

In section 6 of this article we examine when do a few chosen subrings of $C_{c}(X)$ become Noetherian/ Artinian (Theorem 6.4). It follows as special cases that a zero-dimensional space $X$ is finite if and only if $C_{c}(X)$ is Noetherian if and only if $C_{c}(X)$ is Artinian. Furthermore a locally compact zero-dimensional space $X$ is seen to be finite if and only if $C_{c}(X) \cap C_{K}(X)$ becomes Noetherian/Artinian if and only if $C_{c}(X) \cap C_{\infty}(X)$ becomes Noetherian/ Artinian. Here $C_{\infty}(X)$ stands for the rings of all real valued continuous functions on $X$ which vanish at infinity and $C_{K}(X)$ is the subring of $C_{\infty}(X)$ containing those functions which have compact support.

In the final section 7 of this article we give an explicit formula for $z^{0}$-ideals in a typical intermediate ring $A_{c}(X) \in \Sigma_{c}(X)$ (Theorem 7.1). From this it follows that $z^{o}$-ideals of $A_{c}(X)$, in particular $z^{0}$-ideals of $C_{c}(X)$ or $C_{c}^{*}(X)$ are the contraction of $z^{0}$-ideals in $C(X)$.

\section{STRUCTURE SPACES OF INTERMEDIATE RINGS}

We recall from (7M [13]) that if $A$ is a commutative ring with unity and $\mathcal{M}(A)$ the set of all maximal ideals of $\mathrm{A}$ and for each $a \in A$ if we set $\mathcal{M}_{a}=\{M \in \mathcal{M}(A)$ : $a \in M\}$, then the family $\left\{\mathcal{M}_{a}: a \in A\right\}$ turns out to be a closed base for the hull-kernel topology on $\mathcal{M}(A)$. For any $\mathcal{M}_{0} \subseteq \mathcal{M}(A)$ the closure of $\mathcal{M}_{0}=\{M \in$ $\left.\mathcal{M}(A): M \supset \cap \mathcal{M}_{0}\right\} . \mathcal{M}(A)$ equipped with this topology known as the structure space of $A$ is a compact $T_{1}$ topological space and is Hausdorff if and only if given any two distinct maximal ideals $M_{1}, M_{2}$ in $A$, there exist points $a_{1}, a_{2}$ in $A$ such that $a_{1} \notin M_{1}$ and $a_{2} \notin M_{2}$ and $a_{1} a_{2} \in \cap \mathcal{M}(A)$. In what follows we will let $A_{c}(X)$ stand for a typical intermediate ring lying between the two rings $C_{c}^{*}(X)$ and $C_{c}(X)$. Suppose $\operatorname{Max}\left(A_{c}(X)\right)$ denotes the structure space of $A_{c}(X)$.

Theorem 2.1. $\operatorname{Max}\left(A_{c}(X)\right)$ is a (compact) Hausdorff space.

Proof. We shall prove the Hausdorffness of $\operatorname{Max}\left(A_{c}(X)\right)$ only. For any $f \in A_{c}(X)$, set $\mathcal{Z}_{A}(f)=\left\{Z \in Z_{c}(X)\right.$ : there exists $g \in A_{c}(X)$ such that for each $x \in X \backslash$ $Z, f(x) g(x)=1\}$. Here $Z_{c}(X)=\left\{Z(f): f \in C_{c}(X\}\right.$, the family of all zero sets in $X$ of functions lying in $C_{c}(X)$. For any ideal $I$ in $A_{c}(X)$, let $\mathcal{Z}_{A}[I]=\bigcup_{f \in I} \mathcal{Z}_{A}(f)$. Then it can be proved by following the technique adopted in [10, 20, 21] that $\mathcal{Z}_{A}(f)$ and $\mathcal{Z}_{A}[I]$ are both $z_{c}$-filter on $X$. A $z_{c}$-filter on $X$ is a subfamily of $Z_{c}(X)-\{\emptyset\}$ which is closed under finite intersection and formation of supersets (see [14]). Furthermore if $\mathcal{F}$ is a $z_{c}$-filter on $X$, then it can be checked by using the methods in [10, 20, 21] that $\mathcal{Z}_{A}^{-1}[\mathcal{F}]=\left\{f \in A_{c}(X): \mathcal{Z}_{A}(f) \subseteq \mathcal{F}\right\}$ is a (proper) ideal in $A_{c}(X)$. Now let $M_{1}$ and $M_{2}$ be two distinct members of $A_{c}(X)$. It is sufficient to produce $h_{1}, h_{2}$ in $\mathcal{M}_{c}(X)$ with $h_{1} \notin M_{1}, h_{2} \notin M_{2}$ such that $h_{1} h_{2}=0$. To this end we assert that there exists $Z_{1} \in \mathcal{Z}_{A}\left[M_{1}\right], Z_{2} \in \mathcal{Z}_{A}\left[M_{2}\right]$ with $Z_{1} \cap Z_{2}=\emptyset$. For otherwise each member of $\mathcal{Z}_{A}\left[M_{1}\right]$ meets any member of $\mathcal{Z}_{A}\left[M_{2}\right]$ and hence $\mathcal{Z}_{A}\left[M_{1}\right] \cup \mathcal{Z}_{A}\left[M_{2}\right]$ becomes a subfamily of $Z_{c}(X)$ with finite intersection property. Consequently there exists a $z_{c}$-filter $\mathcal{F}$ on $X$ such that $\mathcal{Z}_{A}\left[M_{1}\right] \cup \mathcal{Z}_{A}\left[M_{2}\right] \subseteq \mathcal{F}$ which yields that $M_{1} \cup M_{2} \subseteq$ $\mathcal{Z}_{A}^{-1}[\mathcal{F}]=$ a proper ideal in $A_{c}(X)$, a contradiction since $M_{1}$ and $M_{2}$ are distinct maximal ideals in $A_{c}(X)$. So choose $f \in M_{1}$ and $g \in M_{2}$ such that $Z_{1} \in \mathcal{Z}_{A}(f)$, 
$Z_{2} \in \mathcal{Z}_{A}(g)$ and $Z_{1} \cap Z_{2}=\emptyset$. This means that there exist $f_{1}, g_{1} \in A_{c}(X)$ such that for each $x \in X-Z_{1}, f(x) f_{1}(x)=1$ and for any $x \in X-Z_{2}, g(x) g_{1}(x)=1$. Now since $f f_{1} \in M_{1}$ and $g g_{1} \in M_{2}$, it follows that $1-f f_{1} \notin M_{1}$ and $1-g g_{1} \notin M_{2}$. Since $\left(X-Z_{1}\right) \cup\left(X-Z_{2}\right)=X-\left(Z_{1} \cap Z_{2}\right)=X-\emptyset=X$, it implies that $\left(1-f f_{1}\right) \cdot\left(1-g g_{1}\right)=0$.

We set for any $x \in X, M_{A, x}=\left\{f \in A_{c}(X): f(x)=0\right\}$. Then it is easy to prove on applying the first isomorphism theorem of algebra, taking care of the presence of constant functions in $A_{c}(X)$ that the complete list of fixed maximal ideals of $A_{c}(X)$ is given by $\left\{M_{A, x}: x \in X\right\}$. An ideal $I$ in $A_{c}(X)$ is called fixed if there exists a point on $\mathrm{X}$ at which all the functions in $I$ vanish. For any $f \in A_{c}(X)$ we set $\left(\mathcal{M}_{A}\right)_{f}=\left\{M \in \operatorname{Max}\left(A_{c}(X)\right): f \in M\right\}$. Then $\left\{\left(\mathcal{M}_{A}\right)_{f}: f \in A_{c}(X)\right\}$ is the family of basic closed sets in the structure space $\operatorname{Max}\left(A_{c}(X)\right)$ of $A_{c}(X)$. For $f \in A_{c}(X)$ and $x \in X, x \in Z(f)$ if and only if $M_{A, x} \in(\mathcal{M}(A))_{f} \cap\left\{M_{A, y}: y \in X\right\}$. On the other hand since $X$ is zero-dimensional it follows from Proposition 4 in [5] that $\left\{Z(f): f \in C_{c}^{*}(X)\right\}=\left\{Z(f): f \in A_{c}(X)\right\}=\left\{Z(f): f \in C_{c}(X)\right\}$ constitutes a base for the closed sets of $X$. These two facts therefore yield that the map $\psi_{A}: X \rightarrow \operatorname{Max}\left(A_{c}(X)\right)$ given by $\psi_{A}(x)=M_{A, x}$ which is obviously oneto-one exchanges the basic closed sets of the space $X$ and the subspace $\psi_{A}(X)$ of $\operatorname{Max}\left(A_{c}(X)\right)$. Furthermore the closure of $\psi_{A}(X)$ in $\operatorname{Max}\left(A_{c}(X)\right)$ is given by $\left\{M \in \operatorname{Max}\left(A_{c}(X)\right): M \supseteq \bigcap \psi_{A}(X)\right\}=\left\{M \in \operatorname{Max}\left(A_{c}(X)\right): M \supseteq\{0\}\right\}=$ $\operatorname{Max}\left(A_{c}(X)\right)$, thus demonstrating that $\psi_{A}(X)$ is dense in $\operatorname{Max}\left(A_{c}(X)\right)$. The above observations therefore lead to the following proposition.

Theorem 2.2. The pair $\left(\Psi_{A}, \operatorname{Max}\left(A_{c}(X)\right)\right)$ is a Hausdorff compactification of $X$ in the following sense, which we reproduce from the monograph [12].

Definition 2.3. A (Hausdorff) compactification of a Tychonoff space $X$ stands for a pair $(\alpha, \alpha X)$, where $\alpha X$ is a compact Hausdorff space and $\alpha: X \rightarrow \alpha X$ is a topological embedding with $\alpha(X)$ dense in $\alpha X$. For simplicity we often write $\alpha X$ instead of $(\alpha, \alpha X)$. Let $K(X)$ be the family of all Hausdorff compactifications of $X$.

Definition 2.4. For $\alpha X, \gamma X \in K(X)$, we write $\alpha X \geqq \gamma X$ if there is a continuous map $t: \alpha X \rightarrow \gamma X$ with the property $t \circ \alpha=\gamma$. If in this definition ' $t$ ' is a homeomorphism then we say that $\alpha X$ is topologically equivalent to $\gamma X$ and we write $\alpha X \approx \gamma X$. It can be proved without difficulty that for $\alpha X, \gamma X \in K(X), \alpha X \approx \gamma X$ when and only when $\alpha X \geqq \gamma X$ and $\gamma X \geqq \alpha X$. Furthermore $(K(X), \geqq)$ becomes a complete upper semilattice, which has definitely then a largest member,which is

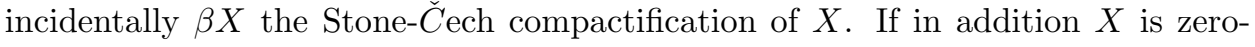
dimensional then there is a largest zero-dimensional member of $K(X)$, designated by $\beta_{0} X$, called the Banaschewski compactification of $X$. For more information on these topics see [19].

Definition 2.5. For a zero-dimensional space $X, \alpha X \in K(X)$ is said to enjoy Cextension property if given any compact Hausdorff zero-dimensional space $Y$ and a continuous map $f: X \rightarrow Y$ there exists a unique continuous map $f^{\alpha}: \alpha X \rightarrow Y$ such that $f^{\alpha} \circ \alpha=f$.

It is clear from the above definition that if $\alpha X \in K(X)$ possesses $C$-extension property then $\alpha X \geqq \beta_{0} X$ and if in addition $\alpha X$ is zero-dimensional then $\beta_{0} X \geqq \alpha X$ 
and consequently $\alpha X \approx \beta_{0} X$. We need the following subsidiary result before stating the first principal technical result of this section.

Theorem 2.6. Let $X$ be zero-dimensional and $A_{c}(X) \in \Sigma_{c}(X)$. Then given $f \in$ $A_{c}(X)$, there exists an idempotent $e$ in $A_{c}(X)$ such that $e$ is multiple of $f$ and $(1-e)$ is a multiple of $(1-f)$ in this ring. [A special case of this result with $A_{c}(X)=C_{c}(X)$ is proved in Remark 3.6 in [5] ].

Proof. There exists $r, 0<r<1$ such that $r \notin f(X)$. Let $W=f^{-1}(-\infty, r)=$ $f^{-1}((-\infty, r])$. So $W$ and $X-W$ are both clopen sets in $X$. The function $e: X \rightarrow R$ defined by the rule : $e(W)=0$ and $e(X-W)=1$ is clearly an idempotent in the ring $A_{c}(X)$. Define the functions $h: X \rightarrow R$ and $k: X \rightarrow R$ as follows: $h(W)=0$ and $h(x)=\frac{1}{f(x)}$ if $x \in X-W \cdot k(X-W)=0$ and $k(x)=\frac{1}{1-f(x)}$ if $x \in W$. Clearly $h$ and $k$ are both bounded functions in $C_{c}(X)$ and hence both are members of the ring $A_{c}(X)$. It is easy to see that $e=h . f$ and $1-e=k(1-f)$.

Theorem 2.7. $\operatorname{Max}\left(A_{c}(X)\right)$ is a (compact Hausdorff) zero-dimensional space. Furthermore the pair $\left(\Psi_{A}, \operatorname{Max}\left(A_{c}(X)\right)\right.$ is topologically equivalent to $\beta_{0} X$. If in addition $X$ is strongly zero-dimensional meaning that $\beta X$ is zero-dimensional, then $\left(\Psi_{A}, \operatorname{Max}\left(A_{c}(X)\right)\right)$ is topologically equivalent to $\beta X$.

Proof. We first prove (only) the zero-dimensionality of $\operatorname{Max}\left(A_{C}(X)\right)$, because of Theorem 2.1. We recall the notation that for any $f \in A_{c}(X),\left(\mathcal{M}_{A}\right)_{f}=\{M \in$ $\left.\operatorname{Max}\left(A_{c}(X)\right): f \in M\right\}$. So let $M \in \operatorname{Max}\left(A_{c}(X)\right)$ and $f \in A_{c}(X)$ be such that $M \in \operatorname{Max}\left(A_{c}(X)\right) \backslash\left(\mathcal{M}_{A}\right)_{f}$. It suffices to find out a clopen set in $\operatorname{Max}\left(A_{c}(X)\right)$ which contains $M$ and is contained in $\operatorname{Max}\left(A_{c}(X)\right) \backslash\left(\mathcal{M}_{A}\right)_{f}$. We first observe that $M \notin\left(\mathcal{M}_{A}\right)_{f}$ implies that $f \notin M$ which in turn implies that there exist $h \in A\left({ }_{c}(X)\right)$ and $g \in M$ such that $1-g=h f$. By Theorem 2.6, there exists an idempotent ' $e^{\prime}$ in $A_{c}(X)$ such that $e$ is a multiple of $g$ and $(1-e)$ is a multiple of $(1-g)$ in the ring $A_{c}(X)$. Since $g \in M$, this implies that $e \in M$, in other words $M \in\left(\mathcal{M}_{A}\right)_{e}$. On the otherhand if $N \in\left(\mathcal{M}_{A}\right)_{f}$ then $f \in N$, hence $1-g=h f \in N$ consequently $1-e \in N$ and therefore $e \notin N$ (as $N$ is a maximal ideal in $A_{c}(X)$ ) which means that $N \notin$ $\left(\mathcal{M}_{A}\right)_{e}$. Thus we get that $M \in\left(\mathcal{M}_{A}\right)_{e} \subseteq \operatorname{Max}\left(A_{c}(X)\right) \backslash\left(\mathcal{M}_{A}\right)_{f}$. We now assert that $\left(\mathcal{M}_{A}\right)_{e}=\operatorname{Max}\left(A_{c}(X)\right) \backslash\left(\mathcal{M}_{A}\right)_{1-e}$ and have $\left(\mathcal{M}_{A}\right)_{e}$ is clopen in $\operatorname{Max}\left(A_{c}(X)\right.$. Indeed if $M \in\left(\mathcal{M}_{A}\right)_{e}$ then $e \in M$, which implies that $1-e \notin M$ and hence $M \notin\left(\mathcal{M}_{A}\right)_{1-e}$ i.e; $M \in \operatorname{Max}\left(A_{c}(X)\right) \backslash\left(\mathcal{M}_{A}\right)_{1-e}$. Thus $\left(\mathcal{M}_{A}\right)_{e} \subseteq \operatorname{Max}\left(A_{c}(X)\right) \backslash$ $\left(\mathcal{M}_{A}\right)_{1-e}$. From symmetry it follows that, as $(1-e)$ is an idempotent of $A_{c}(X)$. $\left(\mathcal{M}_{A}\right)_{1-e} \subseteq \operatorname{Max}\left(A_{c}(X)\right) \backslash\left(\mathcal{M}_{A}\right)_{e}$, hence $\left(\mathcal{M}_{A}\right)_{e}=\operatorname{Max}\left(A_{c}(X)\right) \backslash\left(\mathcal{M}_{A}\right)_{1-e}$.

Now that we have proved that $\operatorname{Max}\left(A_{c}(X)\right)$ is zero-dimensional, to prove the second part of the present theorem, it is sufficient to prove that $\left(\Psi_{A}, \operatorname{Max}\left(A_{c}(X)\right)\right.$ enjoys the $C$-extension property. So let $Y$ be a compact Hausdorff zero-dimensional space and $f: X \rightarrow Y$ a continuous map. It is sufficient to define a continuous map $f^{\mathcal{M}}: \operatorname{Max}\left(A_{c}(X)\right) \rightarrow Y$ with the following property: $f^{\mathcal{M}} \circ \Psi_{A}=f$. To that end choose $M \in \operatorname{Max}\left(A_{c}(X)\right)$ i.e; $M$ is a maximal ideal in $A_{c}(X)$. Set $\widetilde{M}=\left\{g \in C_{c}(Y): g \circ f \in M\right\}$. Note that if $g \in C_{c}(Y)$ then $g \circ f \in C_{c}(X)$. Furthermore since $Y$ is compact and $g \in C_{c}(Y)$ then $g(Y)$ is a bounded subset of $\mathbb{R}$, consequently $(g \circ f)(X)$ is a bounded subset of $\mathbb{R}$ and hence $(g \circ f) \in C_{c}^{*}(X)$ and therefore $g \circ f \in A_{c}(X)$. Thus the definition of $\widetilde{M}$ is without any ambiguity. Since $M$ is a maximal ideal of $A_{c}(X)$ it follows that $\widetilde{M}$ is a prime ideal of $C_{c}(Y)$. Now it is already proved in (14, Corollary 2.14) that every prime ideal in $C_{c}(Y)$ 
is contained in a unique maximal ideal. Thus $\widetilde{M}$ extends to a unique maximal ideal $\operatorname{in} C_{c}(Y)$ which is fixed because $Y$ is compact. Thus there exists a unique point $y \in Y$ such that for each $g \in \widetilde{M}, g(y)=0$ and hence $\bigcap_{g \in \widetilde{M}} Z(g)=\{y\}$. We set $f^{\mathcal{M}}(M)=y$. Thus $\left\{f^{\mathcal{M}}(M)\right\}=\bigcap_{g \in \widetilde{M}} Z(g) \ldots(1)$. We note that if $x \in X$ and $g \in \widetilde{M}_{A, x}$, then $g \circ f \in M_{A, x}$ and hence $(g \circ f)(x)=0$, which implies that $f(x) \in Z(g)$. This proves that $\bigcap Z(g)=\{f(x)\}$. This implies in view of the $g \in \widetilde{M}_{A, x}$

definition (1) above that $f^{\mathcal{M}}\left(M_{A, x}\right)=f(x)$, in other words: $f^{\mathcal{M}} \circ \Psi_{A}(x)=f(x)$. Thus $f^{\mathcal{M}} \circ \Psi_{A}=f$. To ensure the continuity of the map $f^{\mathcal{M}}: \operatorname{Max}\left(A_{c}(X) \rightarrow Y\right.$ defined in (1) at an arbitrary $M \in \operatorname{Max}\left(A_{c}(X)\right)$, let $W$ be a neighbourhood of $f^{\mathcal{M}}(M)$ in the space $Y$. Since $Y$ is zero-dimensional, each neighbourhood of a point ' $y$ ' in this space contains a co-zero set neighbourhood of $y$ of the form $Y \backslash Z\left(g_{1}\right)$ for some $g_{1} \in C_{c}(Y)$ and also a zero set neighbourhood of $y$ of the form $Z\left(g_{2}\right)$ for an appropriate $g_{2} \in C_{c}(Y)$ (see Proposition 4.4, [14]). Thus there exist $g_{1}, g_{2} \in C_{c}(Y)$ such that $f^{\mathcal{M}} \in Y \backslash Z\left(g_{1}\right) \subset Z\left(g_{2}\right) \subset W \ldots(2)$. As $f^{\mathcal{M}}(M) \notin Z\left(g_{1}\right)$, it follows from (1) that $g_{1} \notin \widetilde{M}$ which implies that $g_{1} \circ f \notin M$, in other words $M \notin\left(\mathcal{M}_{A}\right)_{g_{1} \circ f}$. Thus $\operatorname{Max}\left(A_{c}(X)\right) \backslash\left(\mathcal{M}_{A}\right)_{g_{1} \circ f}$ becomes an open neighbourhood of $M$ in the space $\operatorname{Max}\left(A_{c}(X)\right)$. We assert that $\left.f^{\mathcal{M}}\left(\operatorname{Max}\left(A_{c}(X)\right) \backslash\left(\mathcal{M}_{A}\right)_{g_{1} \circ f}\right)\right) \subseteq W$ and this settles the continuity of $f^{\mathcal{M}}$ at the point $M$.

Proof of the last assertion: Let $N \in\left(\operatorname{Max}\left(A_{c}(X)\right) \backslash\left(\mathcal{M}_{A}\right)_{g_{1} \circ f}\right.$, then $g_{1} \circ f \notin N$, hence $g_{1} \notin \tilde{N}$. Since $g_{1} g_{2}=0$ as is evident from the relation (2) above and $\widetilde{N}$ is a prime ideal in $\mathrm{C}(\mathrm{Y})$, it follows therefore that $g_{2} \in \tilde{N}$. This implies in view of the relation (1) that $f^{\mathcal{M}}(N) \in Z\left(g_{2}\right)$ and hence from (2) we get that $f^{\mathcal{M}}(N) \in W$.

The part three of the theorem follows from the simple observation that if $\beta X$ is zero-dimensional, then $\beta_{0} X \geqq \beta X$ and consequently $\beta_{0} X \approx \beta X$.

\section{3. $P$-Spaces $X$ versus the $m_{c}$-Topology on $C_{c}(X)$}

Notation 3.1. For any $g \in C_{c}(X)$ and a positive unit $u$ of this ring set $M(g, u)=$ $\left\{f \in C_{c}(X):|f(x)-g(x)|<u(x)\right.$ for each $\left.x \in X\right\}$. Then it needs a routine calculation to conclude that $\mathcal{B}=\left\{M(g, u): g \in C_{c}(X), u\right.$ a positive unit of $\left.C_{c}(X)\right\}$ is an open base for some topology, which we call the $m_{c}$-topology on $C_{c}(X)$. It is also not at all hard to show by employing stereotyped routine arguments that $C_{c}(X)$ with $m_{c}$-topology is a topological ring as well as a topological vector space over $\mathbb{R}$. Let $U$ stand for the set of all units in $C_{c}(X)$. Then for each $u \in U$, it is easy to prove that $M\left(u, \frac{1}{2}|u|\right) \subseteq U$. It follows that $U$ is an open set in $C_{c}(X)$ in the $m_{c}$-topology. It is a standard result that in a topological ring the closure of an ideal is either an ideal or the whole of the ring (2M1, 13). This implies that if $I$ is a proper ideal of $C_{c}(X)$ then the closure of $I$ in the $m_{c}$-topology is also a proper ideal in $C_{c}(X)$. We therefore get the following result:

Theorem 3.2. Each maximal ideal in $C_{c}(X)$ is closed in the $m_{c}$-topology.

Before proceeding further in this technical section on $m_{c}$-topology on $C_{c}(X)$ we recall that the structure space of $C_{c}(X)$ is $\beta_{0} X$. Hence the maximal ideals of $C_{c}(X)$ can be indexed by virtue of the points of $\beta_{0} X$. Indeed the complete list of maximal ideals in $C_{c}(X)$ is given in ([5], Theorem 4.2) by the family $\left\{M_{c}^{p}: p \in \beta_{0} X\right\}$, where 
$M_{c}^{p}=\left\{f \in C_{c}(X): p \in c l_{\beta_{0} X} Z(f)\right\}$. This is the $C$-analogue of the well known Gelfand-Kolmogoroff theorem ([13, Theorem 7.3).

Notation 3.3. For any ideal $I$ in $C_{c}(X)$ set $Q_{c}(I)=\left\{p \in \beta_{0} X: M_{c}^{p} \supseteq I\right\}$. Then the following result turns out as a simple consequence of the above formula for the maximal ideals $M_{c}^{p}$ 's in $C_{c}(X)$.

Theorem 3.4. $Q_{c}(I)=\bigcap_{f \in I} c l_{\beta_{0} X} Z(f)$, which is set of all cluster points of the $z_{c}$-ultrafilters $Z(I)$ in the space $\beta_{0} X$.

We need to use the following three subsidiary results to prove the first important technical result in this section.

Theorem 3.5. Let $f \in C_{c}(X)$ and $I$ be an ideal in $C_{c}(X)$ such that $c_{\beta_{0} X} Z(f)$ is a neighbourhood of $Q_{c}(I)$ in $\beta_{0} X$. Then $f \in I$.

Proof. The hypothesis tells that there exists an open subset $W$ of $\beta_{0} X$ such that $c l_{\beta_{0} X} Z(f) \supseteq W \supseteq Q_{c}(I)$. We can rewrite this relation in view of Theorem 3.4 in the manner: $\operatorname{cl}_{\beta_{0} X} Z(f) \supseteq W \supseteq \bigcap_{f \in I} c l_{\beta_{0} X} Z(f)$. This implies that $\beta_{0} X \backslash c l_{\beta_{0} X} Z(f) \subseteq$ $\beta_{0} X \backslash W \subseteq \bigcup_{f \in I}\left(\beta_{0} X \backslash c l_{\beta_{0} X} Z(f)\right)$. Since the closed subset $\beta_{0} X \backslash W$ of $\beta_{0} X$ is compact, the last relation yields : $\beta_{0} X \backslash c l_{\beta_{0} X} Z(f) \subseteq \beta_{0} X \backslash W \subseteq \beta_{0} X \backslash \bigcap_{i=1}^{n} c l_{\beta_{0} X} Z\left(f_{i}\right)$ for a suitable finite subset $\left\{f_{1}, f_{2} \ldots f_{n}\right\}$ of $I$. Consequently we have $\operatorname{cl}_{\beta_{0} X} Z(f) \supseteq W \supseteq$ $\bigcap_{i=1}^{n} c l_{\beta_{0} X} Z\left(f_{i}\right)$, which further implies that $c l_{\beta_{0} X} Z(f) \cap X \supseteq W \cap X \supseteq Z\left(\sum_{i=1}^{n} f_{i}^{2}\right)=$ $Z(h)$ say, writing $h=f_{1}^{2}+f_{2}^{2}+\ldots+f_{n}^{2}$. The last relation says that with $h \in I$, $Z(f)$ is a neighbourhood of $Z(h)$ in the space $X$. It follows from Lemma 2.4 in [14] that $f$ is a multiple of $h$ in the $\operatorname{ring} C_{c}(X)$. Since $h \in I$, we have $f \in I$.

Theorem 3.6. Given $g \in C_{c}(X)$ and a positive unit $u$ in this ring, there exists $f \in C_{c}(X)$ such that $|g-f| \leq u$ and $c_{\beta_{0} X} Z(f)$ is a neighbourhood of $c_{\beta_{0} X} Z(g)$ in the space $\beta_{0} X$.

Proof. Let the map $f: X \rightarrow \mathbb{R}$ be defined as follows:

$$
f(x)= \begin{cases}0 & \text { if }|g(x)| \leq u(x) \\ g(x)+u(x) & \text { if } g(x) \leq-u(x) \\ g(x)-u(x) & \text { if } g(x) \geq u(x)\end{cases}
$$

It is clear that $f$ is a continuous function and of course $f \in C_{c}(X)$. It is easily seen that $|f-g| \leq u$ on $X$. Let $F=\{x \in X:|g(x)| \geq u(x)\}$. Then $F \in Z_{c}(X)$ so that we can write $F=Z_{c}(h)$ for some $h \in C_{c}(X)$. Hence $Z(g) \subseteq X \backslash Z(h) \subseteq Z(f)$. This implies that $Z(g) \cap Z(h)=\emptyset$ and $Z(f) \cup Z(h)=X$. From this it follows that $c l_{\beta_{0} X} Z(g) \cap c l_{\beta_{0} X} Z(h)=\emptyset$ and $c l_{\beta_{0} X} Z(g) \cup c l_{\beta_{0} X} Z(f)=c l_{\beta_{0} X} X=\beta_{0} X$ [see Proposition 3.2 and Proposition 3.3 in [5]]. This further yields:

$c l_{\beta_{0} X} Z(f) \supseteq \beta_{0} X \backslash c l_{\beta_{0} X} Z(h) \supseteq c l_{\beta_{0} X} Z(g)$ this shows that $c_{\beta_{0} X} Z(f)$ is a neighbourhood of $c l_{\beta_{0} X} Z(g)$ in the space $\beta_{0} X$.

Define as in 7Q [13, for an ideal $I$ in $C_{c}(X)$. $\bar{I}=\bigcap\left\{M_{c}^{p}: M_{c}^{p} \supseteq I\right\}=$ the intersection of all maximal ideals in $C_{c}(X)$ which contain $I$. 
Theorem 3.7. For any ideal $I$ in $C_{c}(X) \bar{I}$ is a closed ideal in the $m_{c}$-topology and $\bar{I}=\left\{g \in C_{c}(X): c l_{\beta_{0} X} Z(g) \supseteq Q_{c}(I)\right\}$.

Proof. It follows immediately from Theorem 3.2 that $\bar{I}$ is a closed ideal in $C_{c}(X)$ in the $m_{c}$-topology. Let $g \in \bar{I}$, choose $x \in Q_{c}(I)$, then $M_{c}^{x} \supseteq I$. Consequently $g \in M_{c}^{x}$ and hence $x \in c l_{\beta_{0} X} Z(g)$. This implies that $Q_{c}(I) \subseteq c_{\beta_{0} X} Z(g)$. To prove the reverse inclusion relation let $g \in C_{c}(X)$ be such that $Q_{c}(I) \subseteq c l_{\beta_{0} X} Z(g)$. Let $M_{c}^{p}$ be any maximal ideal in $C_{c}(X)$ containing $I, p \in \beta_{0} X$. Then $p \in Q_{c}(I)$ consequently $p \in \operatorname{cl}_{\beta_{0} X} Z(g)$ hence $g \in M_{c}^{p}$. Thus $g \in \bar{I}$.

Theorem 3.8. For any ideal $I$ in $C_{c}(X), \bar{I}$ is essentially the closure of $I$ in the $m_{c}$-topology.

Proof. It follows from the first part of Theorem 3.7 that the closure of $I$ in the $m_{c^{-}}$ topology is contained in $\bar{I}$. To prove the reverse containment let $g \in \bar{I}$ and $u$ be a positive unit of $C_{c}(X)$. It suffices to produce an $h \in I$ such that $|g-h| \leq u$. Indeed from Theorem 3.6 there exists an $h \in C_{c}(X)$ with $|g-h| \leq u$ such that $c l_{\beta_{0} X} Z(h)$ is a neighbourhood of $c l_{\beta_{0} X} Z(g)$ in the space $\beta_{0} X$. But $g \in \bar{I}$ implies by Theorem 3.7 that $c l_{\beta_{0} X} Z(g) \supseteq Q_{c}(I)$. Consequently $c l_{\beta_{0} X} Z(h)$ becomes a neighbourhood of $Q_{c}(I)$ in $\beta_{0} X$. Hence we get from Theorem 3.5 that $h \in I$.

Corollary 3.9. An ideal in $C_{c}(X)$ is closed in the $m_{c}$-topology if and only if it is the intersection of all the maximal ideals in $C_{c}(X)$ which contain it.

Theorem 3.10. A zero-dimensional space $X$ is a $P$-space if and only if each ideal in $C_{c}(X)$ is closed in the $m_{c}$-topology.

Proof. It follows from Corollary 3.9 that each ideal $I$ in $C_{c}(X)$ is closed in the $m_{c}$-topology if and only if each ideal in $C_{c}(X)$ is the intersection of all the maximal ideals in $C_{c}(X)$ containing it. In view of Corollary 5.7 and Theorem 5.8 in [14, the last condition is equivalent to the requirement that $X$ is a $P$-space.

Before examining the Von-Neumann regularity of the intermediate rings in the family $\Sigma_{c}(X)$, we need to further organize our machinery accordingly. A commutative ring $R$ with unity is called reduced if 0 is the only nilpotent element of $R$. It is trivial that each $A_{c}(X) \in \Sigma_{c}(X)$ is a reduced ring. In what follows all the rings that will appear will be assumed to be reduced. An ideal $I$ (proper) in $R$ is called a $z^{0}$-ideal in $R$ if for each $a \in I, \mathcal{P}_{a} \subseteq I$, where $\mathcal{P}_{a}$ is the intersection of all minimal prime ideals in $R$ which contains a. We reproduce the following standard useful formula for the $\mathcal{P}_{a}$ from ([7, Proposition 1.5).

Theorem 3.11. For each $a \in R, \mathcal{P}_{a}=\{b \in R:$ Ann $(a) \subseteq$ Ann $(b)\}$, where Ann $(a)=\{c \in R: a c=0\}$ is the annihilator of $a$ in $R$. We also reproduce the following standard proposition.

Theorem 3.12. (Due to Kist, 17]): A prime ideal $P$ in a ring $R$ is a minimal prime ideal if and only if for each $a \in P$ there exists $b \in R \backslash P$ such that a.b is a nilpotent member of $R$ and in particular $a . b=0$ if the ring $R$ is assumed to to reduced.

Remark 3.13. Each element of a minimal prime ideal in $R$ is a divisor of zero. Consequently each element of a $z^{0}$-ideal in $R$ is a divisor of zero.

The following fact is standard and a simple proof is offered in [9] Theorem 4.1. 
Theorem 3.14. Each proper ideal in a Von-Neumann regular ring is a $z^{0}$-ideal.

Theorem 3.15. An intermediate ring $A_{c}(X) \in \Sigma_{c}(X)$ is an absolutely convex subring of $C_{c}(X)$ in the following sence: If $|f| \leq|g|$ with $g \in A_{c}(X)$ and $f \in C_{c}(X)$ then $f \in A_{c}(X)$. In particular $A_{c}(X)$ is a lattice ordered ring.

Proof. since If $|f| \leq|g|$ it follows that $\frac{f}{1+g^{2}}$ is a bounded function in $C_{c}(X)$. Thus $f=\frac{f}{1+g^{2}} \cdot\left(1+g^{2}\right) \in A_{c}(X)$.

The following result tells that no intermediate ring in the family $\Sigma_{c}(X) \backslash\left\{C_{c}(X)\right\}$ can be ever Von-Neumann regular.

Theorem 3.16. Suppose $A_{c}(X) \in \Sigma_{c}(X)$ is Von-Neumann regular, then $A_{c}(X)=$ $C_{c}(X)$.

Proof. Choose $f \in C_{c}(X)$. We shall show that $f \in A_{c}(X)$. Because of the absolute convexity of $A_{c}(X)$ in $C_{c}(X)$ in the last theorem it suffices to show that $|f| \in A_{c}(X)$. We shall indeed show that $\frac{1}{1+|f|}$ is a multiplicative unit of the ring $A_{c}(X)$ and that will do. Suppose towards a contradiction and let $\frac{1}{1+|f|}$ be not a multiplicative unit of $A_{c}(X)$. It is clear because of the boundedness of the function $\frac{1}{1+|f|}$ over $X$ that $\frac{1}{1+|f|} \in A_{c}(X)$. Therefore the principle ideal $<\frac{1}{1+|f|}>=I$ in $A_{c}(X)$ generated by this function is a proper ideal and is hence by Theorem $3.14 \mathrm{a} z^{0}$ ideal in $A_{c}(X)$. It follows from Remark 3.13 that $\frac{1}{1+|f|}$ is a divisor of zero in $A_{c}(X)$ -a contradiction.

Since a zero-dimensional space $X$ is a $P$-space if and only if $C_{c}(X)$ is VonNeumann regular (Corollary 5.7, [14), the following proposition is immediate from the above theorem.

Theorem 3.17. Let $X$ be a P-space. Then $A_{c}(X) \in \Sigma_{c}(X)$ is Von-Neumann reular if and only if $A_{c}(X)=C_{c}(X)$.

\section{Almost $P$-spaces $X$ Vis-a-vis the $z^{0}$-ideals in $A_{c}(X)$.}

Since the $z^{0}$-ideals in $A_{c}(X)$ are all divisors of zero, the following formula to determine them will be needed from time to time.

Theorem 4.1. An $f \in A_{c}(X)$ is a divisor of zero in this ring if and only if Int $_{X} Z(f) \neq \emptyset$.

Proof. Suppose $f \in A_{c}(X)$ is a divisor of zero. Then $f \neq 0$ and there exists $g \neq 0$ in $A_{c}(X)$ such that $f g=0$. This shows that $Z(f) \cup Z(g)=X$ and hence $X-Z(g) \subseteq Z(f)$. As $X \backslash Z(g)$ is a non-empty open set in $X$, it follows that Int $_{X} Z(f) \neq \emptyset$.

Conversely let $\operatorname{Int}_{X} Z(f) \neq \emptyset$. Choose $p$ from this nonempty set. Since $X$ is zerodimensional, functions in $C_{c}(X)$ with their range contained in $[0,1]$ can separate points and closed sets in $X$ (Proposition 4.4, [14]). Therefore there exists $g \in C_{c}(X)$ such that $g(p)=1$ and $g\left(X \backslash \operatorname{Int}_{X} Z(f)\right)=0$. It is clear that $f . g=0$ and $g \neq 0$. Thus $f$ is divisor of zero in $A_{c}(X)$.

The next proposition will also be useful to us: 
Theorem 4.2. Let $X$ be zero-dimensional and $f, g \in A_{c}(X)$. Then $\operatorname{Int}_{X} Z(f) \subseteq$ Int $_{X} Z(g)$ if and only if $A n n(f) \subseteq A n n(g)$ in the ring $A_{c}(X)$.

Proof. Let $\operatorname{Int}_{X} Z(f) \subseteq \operatorname{Int}_{X} Z(g)$. Choose $h \in A n n(f)$, then $h g=0$. This implies that $X \backslash Z(h) \subseteq Z(f)$, which further implies that $X \backslash Z(h) \subseteq \operatorname{Int}_{X} Z(f) \subseteq$ Int $_{X} Z(g) \subseteq Z(g)$. Hence $g . h=0$ i.e., $h \in A n n(g)$. Thus $A n n(f) \subseteq A n n(g)$.

Conversely let $A n n(f) \subseteq A n n(g)$. It is sufficient to check that $I_{n} t_{X} Z(f) \subseteq Z(g)$. If possible let there exist a point $p \in \operatorname{Int}_{X} Z(f) \backslash Z(g)$. Since $X$ is zero-dimensional, there exists an $h \in C_{c}^{*}(X) \subseteq A_{c}(X)$ such that $h(p)=1$ and $h\left(X \backslash \operatorname{Int}_{X} Z(f)\right)=0$. It follows that $h . f=0$ i.e., $h \in A n n(f)$ but $h(p) g(p) \neq 0$. So that $h . g \neq 0$ and hence $h \notin \operatorname{Ann}(g)$. This is a contradiction.

A combination of Theorem 3.10 and Theorem 4.2 yields the following result:

Theorem 4.3. For any $f \in A_{c}(X) \mathcal{P}_{f}=\left\{g \in A_{c}(X): A n n(f) \subseteq A n n(g)\right\}=\{g \in$ $\left.A_{c}(X): \operatorname{Int}_{X} Z(f) \subseteq \operatorname{Int}_{X} Z(g)\right\}$.

We recall that $\mathcal{P}_{f}$ is the intersection of all the minimal prime ideals in $A_{c}(X)$ which contain $f$. Before taking up the problem of characterizing almost $P$-spaces $X$ via $z^{0}$-ideals in $C_{c}(X)$, we need to recall the notion of $z$-ideal in an arbitrary commutative ring $R$ with unity.

Definition 4.4. An ideal $I$ in $R$ is called a $z$-ideal in $R$ if for each $a \in I, M_{a} \subseteq I$, here $M_{a}$ is the intersection of all maximal ideals in $R$ containing $a$. Evidently each maximal ideal in $R$ is a $z$-ideal. This notion of $z$-ideal is consistent with the notion of $z$-ideals in $C(X)$. (See $4 \mathrm{~A},[13$ )

The following result identifies $z$-ideals and $z_{c}$-ideals in $C_{c}(X)$. An ideal $I$ in $C_{c}(X)$ is called a $z_{c}$-ideal in [14] if whenever $Z(f) \in Z_{c}(I)=\{Z(g): g \in I\}, f \in$ $C_{c}(X)$, then $f \in I$.

Theorem 4.5. Let $X$ be zero-dimensional. Then an ideal $I$ in $C_{c}(X)$ is a z-ideal if and only if it is a $z_{c}$-ideal.

Proof. Let $I$ be a $z_{c}$-ideal in $C_{c}(X)$. Let $f \in I$ and $g \in M_{f}$, this means that if for $p \in \beta_{0} X, f \in M_{c}^{p}$ then $g \in M_{c}^{p}$. This implies that $c l_{\beta_{0} X} Z(f) \subseteq c l_{\beta_{0} X} Z(g)$, which further implies on taking intersection with $X$ that $Z(f) \subseteq Z(g)$. Since $f \in I$ and $I$ is a $z_{c}$-ideal in $C_{c}(X)$ it follows that $g \in I$. Thus $M_{f} \subseteq I$ and hence $I$ is a $z$-ideal in $C_{c}(X)$.

Conversely let $I$ be a $z$-ideal in $C_{c}(X), f \in I$ and $Z(f) \subseteq Z(g)$ with $g \in C_{c}(X)$. We have to show that $g \in I$. Since $I$ is a $z$-ideal in $C_{c}(X)$ it suffices to show that $g \in M_{f}$. So let $M_{c}^{p}$ be any maximal ideal in $C_{c}(X)$ it suffices to show that $g \in M_{f}$. So let $M_{c}^{p}$ be any maximal ideal in $C_{c}(X), p \in \beta_{0} X$ which contains $f$, we have to show that $g \in M_{c}^{p}$. Indeed $f \in M_{c}^{p}$ implies that $p \in c l_{\beta_{0} X} Z(f)$ which further implies that $p \in \operatorname{cl}_{\beta_{0} X} Z(g)$ hence $g \in M_{c}^{p}$. Thus altogether $I$ becomes a $z_{c}$-ideal in $C_{c}(X)$.

We next establish the countable analogue of the well-known fact 3.11(b) in [13].

Theorem 4.6. Let $K$ be a compact set contained in a $G_{\delta}$-set $G$ in a zero-dimensional Hausdorff space $X$. Then there exists $Z \in Z_{c}(X)$ such that $K \subseteq Z \subseteq G$.

Proof. We can write $G=\bigcap_{n=1}^{\infty} G_{n}$, where each $G_{n}$ is open in $X$. Since $K \subset X$ and $K \backslash G_{n}$ are disjoint closed set in $X$ with $K$ compact, hence by proposition 4.3 in [14], 
there exists an $f_{n} \in C_{c}(X)$ such that $f_{n}(K)=0$ and $f \in\left(X \backslash G_{n}\right)=1$. This implies that $K \subseteq \bigcap_{n=1}^{\infty} Z\left(f_{n}\right) \subseteq G$. Since $Z_{c}(X)$ is closed under countable intersection by Lemma $2.2(\mathrm{a})$ in [5], it follows that $\bigcap_{n=1}^{\infty} Z\left(f_{n}\right)=Z(f)$ for some $f \in C_{c}(X)$. This implies that $K \subseteq Z(f) \subseteq G$.

Before seriously embarking on almost $P$-spaces, we introduce the following localized version of this requirement.

Definition 4.7. A point $p \in X$ is called an almost $P$-point on $X$ if for any zero set $Z$ in $X$ containing $p, \operatorname{Int}_{X} Z \neq \emptyset$. Thus $X$ is an almost $P$-space if and only if each point on $X$ is an almost $P$-point.

Theorem 4.8. The following statements are equivalent for a point $p$ on a zerodimensional Hausdorff space $X$.

(1) $p$ is an almost $P$-point on $X$.

(2) For any any $G_{\delta}$-set $G$ containing $p, \operatorname{Int}_{X} G \neq \emptyset$.

(3) For any $Z \in Z_{c}(X)$ containing $p, \operatorname{Int}_{X} Z \neq \emptyset$.

Proof. (1) $\Longrightarrow(3)$ and (2) $\Longrightarrow(1)$ are trivial.

$(3) \Longrightarrow(2)$ : Let $(3)$ hold. Let $G$ be a $G_{\delta}$ set in $X$ containing $p$. Then by Theorem 4.6. there exists $Z \in Z_{c}(X)$ such that $p \in Z \subset G$. Since $\operatorname{Int}_{X} Z \neq \emptyset$ it follows from (3) that $\operatorname{Int}_{X} G \neq \emptyset$.

Corollary 4.9. A zero-dimensional space $X$ is an almost $P$-space if and only if for any nonempty $Z \in Z_{c}(X), \operatorname{Int}_{X} Z \neq \emptyset$.

We are now ready to offer the following comprehensive theorem giving several characterization of almost $P$-space.

Theorem 4.10. The following statements are equivalent for a zero-dimensional space $X$.

(1) $X$ is almost $P$.

(2) Every maximal ideal in $C_{c}(X)$ is a $z^{0}$-ideal.

(3) Every fixed maximal ideal in $C_{c}(X)$ is a $z^{0}$-ideal.

(4) Every $z$-ideal in $C_{c}(X)$ is a $z^{0}$-ideal.

Proof. (1) $\Longrightarrow$ (2): Let $X$ be almost $P$-space and $M$ be a maximal ideal in $C_{c}(X)$ - Then we can write $M=M_{c}^{p}=\left\{f \in C_{c}(X): p \in c l_{\beta_{0} X} Z(f)\right\}$ for some point $p \in \beta_{0} X$. Choose $f \in M$ we shall show that $\mathcal{P}_{f} \subseteq M$ and hence $M$ is a $z^{0}$-ideal in $C_{c}(X)$. So let $g \in \mathcal{P}_{f}$. Then from Theorem 4.3 we get $\operatorname{Int}_{X} Z(f) \subseteq \operatorname{Int}_{X} Z(g)$. But since $X$ is almost $P$, each zero set in $X$ is regular closed see [18. This implies that $Z(f)=c l_{X}\left(\operatorname{Int}_{X} Z(f)\right) \subseteq c l_{X}\left(\operatorname{Int}_{X} Z(g)\right)=Z(g)$. But $f \in M$ implies that $p \in c l_{\beta_{0} X} Z(f)$, consequently $p \in c l_{\beta X} Z(g)$ and hence $g \in M_{c}^{p}=M$. Thus $\mathcal{P}_{f} \subseteq M$. $(2) \Longrightarrow(3)$ : is trivial.

$(3) \Longrightarrow(1)$ : Let $(3)$ be true. It is sufficient to show in view of Corollary 4.9 that, for a non-empty $Z \in Z_{c}(X), \operatorname{Int}_{X} Z \neq \emptyset$. Indeed $Z=Z(f)$ for some $f \in C_{c}(X)$. Choose a point $p \in Z$, then $f \in M_{p, c}=\left\{g \in C_{c}(X): g(p)=0\right\}$. Now by $(3), M_{p, c}$ is a $z^{0}$-ideal, consequently by Remark 3.13, $f$ is a divisor of zero in $C_{c}(X)$. This implies by Theorem 4.1 that , $\operatorname{Int}_{X} Z(f) \neq \emptyset$.

$(4) \Longrightarrow(2)$ : is trivial because each maximal ideal in a ring $R$ is a $z$-ideal. 
$(1) \Longrightarrow$ (4): Let $X$ be almost $P$-space and $I$ be a $z$-ideal in $C_{c}(X)$. Then by Theorem 4.5. $I$ is a $z_{c}$-ideal in $C_{c}(X)$. Let $f \in I$ we need to verify that $\mathcal{P}_{f} \subseteq I$ in order to show that $I$ is a $z^{0}$-ideal in $C_{c}(X)$. Choose $g \in \mathcal{P}_{f}$ then it follows from Theorem 4.3 that $\operatorname{Int}_{X} Z(f) \subseteq \operatorname{Int}_{X} Z(g)$. As $X$ is almost $P$ we can therefore write: $Z(f)=c l_{X}\left(\operatorname{Int}_{X} Z(f)\right) \subseteq c l_{X}\left(\operatorname{Int}_{X} Z(g)\right)=Z(g)$. Since $f \in I$ and $I$ is a $z_{c}$-ideal, it follows that $g \in I$. Thus $\mathcal{P}_{f} \subseteq I$.

We now show that on choosing $A_{c}(X) \in \Sigma_{c}(X) \backslash\left\{C_{c}(X)\right\}$ Theorem 4.10 can not be improved by writing that $X$ is almost $P$ if and only if each maximal ideal in $A_{c}(X)$ is a $z^{0}$-ideal (respectively each $z$-ideal in $A_{C}(X)$ is a $z^{0}$-ideal).

Theorem 4.11. Let $A_{c}(X)$ be an intermediate ring in $\Sigma_{c}(X)$ properly contained in $C_{c}(X)$. Then there exists a maximal ideal $M$ in $A_{c}(X)$ which is not a $z^{0}$-ideal (clearly $M$ is also a $z$-ideal in $A_{c}(X)$ which is not a $z^{0}$-ideal).

Proof. We select $f \in C_{c}(X)$ such that $f \notin A_{c}(X)$. Take $g=\frac{1}{1+|f|}$, then $g \in$ $C_{c}^{*}(X) \subseteq A_{c}(X)$. It follows from absolute convexity of $A_{c}(X)$ in $C_{c}(X)$ (Theorem 3.15) that $1+|f| \notin A_{c}(X)$. Hence $g$ is not invertible in $A_{c}(X)$. So there exists a maximal ideal $M$ in $A_{c}(X)$ such that $g \in M$. Since $g$ is not a divisor of zero in $A_{c}(X)$ (Theorem 4.1). It follows from Remark 3.13 that $M$ is not a $z^{0}$-ideal in $A_{c}(X)$.

Theortem 4.10 and Theorem 4.11 combined together yield the following characterization of $C_{c}(X)$ among members of $\Sigma_{c}(X)$.

Theorem 4.12. Let $X$ be almost $P$. Then the following three statements are equivalent for an $A_{c}(X) \in \Sigma_{c}(X)$.

(1) Each maximal ideal of $A_{c}(X)$ is a $z^{0}$-ideal

(2) Each $z$-ideal of $A_{c}(X)$ is a $z^{0}$-ideal

(3) $A_{c}(X)=C_{c}(X)$.

Compare with similar kind of characterizations in 9,22, , 23].

5. Pseudocompact spaces $X$ via $U_{c^{-T O P O l O G i e s}} / m_{c}$-Topologies on $C_{c}(X)$

Notation 5.1. For $f \in C_{c}(X)$ and $\epsilon>0$ in $\mathbb{R}$. Let $U_{c}(f, \epsilon)=\left\{g \in C_{c}(X)\right.$ : $\left.\operatorname{Sup}_{x \in X}|f(x)-g(x)|<\epsilon\right\}$

It is easy to check that the family $\left\{U_{c}(f, \epsilon): f \in C_{c}(X), \epsilon>0\right\}$ is an open base for some topology on $C_{c}(X)$ which we call the $U_{c}$-topology on $C_{c}(X)$ and $C_{c}(X)$ becomes an additive topological group in this topology. The following proposition shows that $C_{c}(X)$ neither a topological ring nor a topological vector space unless $X$ is pseudocompact.

Theorem 5.2. For a zero-dimensional Hausdorff space $X$, the following statements are equivalent:

(1) $X$ is pseudocompact.

(2) $C_{c}(X)$ with $U_{c}$-topology is a topological ring.

(3) $C_{c}(X)$ with $U_{c}$-topology is a topological vector space.

(4) The set $W$ of all units in $C_{c}(X)$ is open in $U_{C}$-topology.

Proof. First assume that $X$ is pseudocompact ie; $C_{c}(X)=C_{c}^{*}(X)$. Then the $U_{C^{-}}$ topology on $C_{c}(X)$ coincides with the uniform norm topology on it and $C_{c}(X)$ 
becomes a real normed algebra. It is a standard result in Functional Analysis that a real normed algebra is a topological ring as well as a real topological vector space, where the units $W$ make an open subset of $C_{c}(X)$. Conversely, let $X$ be not pseudocompact. Then there exists an $f \in C_{c}(X) \backslash C_{c}^{*}(X)$ with $f \geqq 1$. Take $g=\frac{1}{f}$. Then $g \in C_{c}^{*}(X)$ and it takes values arbitrarily near to zero on $X$. We note that for arbitrary $\epsilon>0, \delta>0$ in $\mathbb{R}, \frac{\epsilon}{2}$ (the constant function on $X$ with value $\left.\frac{\epsilon}{2}\right) \in U_{c}(0, \epsilon)$ and $f \in U_{c}(f, \delta)$ while $\frac{\epsilon}{2} . f \notin U_{c}(0,1)$. This proves that the function: $C_{C}(X) \times C_{c}(X) \rightarrow C_{C}(X)$ defined as follows $(k, l) \mapsto k . l$ is not continuous at the point $(0, f)$. It can be proved analogously that the scalar multiplication function:

$$
\begin{gathered}
\mathbb{R} \times C_{c}(X) \rightarrow C_{c}(X) \\
(r, f) \mapsto r . f
\end{gathered}
$$

is not continuous at $(0, f)$. Thus $C_{c}(X)$ neither a topological ring nor a topological vector space over $R$.

Finally we observe that $g$ is a unit of $C_{c}(X)$ i.e; $g \in W$. To show that $W$ is not an open set we shall show that $g$ is not an interior point of $W$. Choose $\epsilon>0$ in $\mathbb{R}$. Since $g$ takes values arbitrarily near to zero on $X$, there exists $a \in X$ such that $0<g(a)<\epsilon$. Take $h=g-g(a)$, then $h \in C_{c}(X)$ and $h \in U_{c}(g, \epsilon)$ but $h$ is not a unit of $C_{c}(X)$ as $h(a)=0$. Thus $U_{c}(g, \epsilon)$ is not a subset of $W$ and hence $g$ is not an interior point of $W$.

As in the classical scenario with $C(X)$ (see 2N, 13]) it is easy to observe that the relative topology on $C_{c}^{*}(X)$ induced by the $m_{c}$-topology on $C_{c}(X)$ is finer that the uniform norm topology on $C_{c}^{*}(X)$. The following proposition says that these two topologies coincide when and only when $X$ is pseudocompact.

Theorem 5.3. The following two statements are equivalent for a Hausdorff zerodimensional space $X$.

(1) $X$ is pseudocompact.

(2) The relative $m_{c}$-topology on $C_{c}^{*}(X)$ is identical to the uniform norm topology on it.

Proof. First assume that $X$ is pseudocompact. In view of the above observations, it is sufficient to show that that relative $m_{c}$-topology $C_{c}^{*}(X)$ is weaker than the uniform norm topology. Choose $f \in C_{c}^{*}(X)$ and a positive unit $u$ of this ring. Then $u$ is bounded away from zero so that we can write $u(x) \geq \lambda$ for all $x \in X$ for some $\lambda>0$. It follows that the closed ball $\left.\left\{g \in C_{c}^{*}(X)\right):\|f-g\| \leq \lambda\right\}$ centered at $f$ with radius $\lambda$ in the norm topology is contained in $M(f, u)$ and we are through.

To prove the converse let $X$ be not pseudocompact. To show that the relative $m_{c}$-topology on $C_{c}^{*}(X)$ is not the same as the uniform norm topology on it, we shall show that $C_{c}^{*}(X)$ in the former topology is not a topological vector space, Since $X$ is not pseudocompact there exists $k \in C_{c}^{*}(X)$ such that $k$ is a positive unit of $C_{c}(X)$ which takes values arbitrarily near to zero on X. It follows that there does not exist any pair of distinct real numbers $r, s$ with $|\underline{r}-\underline{s}| \leq k$ on $X$. Hence for any $r \in \mathbb{R}, M(\underline{r}, k) \cap\{\underline{s}: s \in \mathbb{R}\}=\{\underline{r}\}$ in other words the set of all constant functions in $C_{c}^{*}(X)$ is a discrete subset of $C_{c}^{*}(X)$ in the relative $m_{c}$-topology. Consequently the scalar multiplication map: $\left.\mathbb{R} \times C^{*}(X) \rightarrow C^{*}(X)\right)$ defined as follows $(r, f) \rightarrow r . f$ is not continuous at the points like $(r, \underline{s})$ with $r, s \in \mathbb{R}$, here $\underline{s}$ stands for for the constant function with value ' $s$ ' on $X$. 


\section{Questions of Noetherianness/ Artinianness about $C_{c}(X)$ and their CHOSEN SUBRINGS.}

A commutative ring $R$ (with or without identity) is called Noetherian/ Artinian if any ascending sequence of ideals $I_{1} \subseteq I_{2} \subseteq \ldots$.../ descending sequence of ideals $I_{1} \supseteq I_{2} \supseteq \ldots$. . terminates at a finite stage. It is established in $[2]$ that for a Tychonoff space $X, C(X)$ (respectively $C^{*}(X)$ ) is never Noetherian and also never Artinian unless $X$ is a finite set. Noetherianness/ Artinianness of a selected class of subrings of $C(X)$ are also examined in [2]. In the present section our intention is to record the appropriate counterparts of the problems dealt in [2] in the context of the rings $C_{c}(X)$ and $C_{c}^{*}(X)$ for a zero-dimensional Hausdorff space $X$.

A family $\mathcal{P}$ of closed set in $X$ is called an ideal of closed sets if

(1) $A \in \mathcal{P}, B \in \mathcal{P} \Longrightarrow A \cup B \in \mathcal{P}$ and

(2) $A \in \mathcal{P}$ and $K \subseteq A$ with $K$ closed in $X \Longrightarrow K \in \mathcal{P}$

Notation 6.1. Let $\Omega(X)$ stand for the family of all ideals of closed sets in $X$ with $\mathcal{P} \in \Omega(X)$. We associate the following two subrings of $C(X)$ :

$C_{\mathcal{P}}(X)=\left\{f \in C(X): c l_{X}(X \backslash Z(f)) \in \mathcal{P}\right\}$ and

$C_{\infty}^{\mathcal{P}}(X)=\left\{f \in C(X)\right.$ : for each $\left.n \in N,\left\{x \in X:|f(x)| \geqq \frac{1}{n}\right\} \in \mathcal{P}\right\}$ with $C_{\mathcal{P}}(X)$ a $z$ ideal in $C(X) . X$ is called locally $\mathcal{P}$ if each point $x \in X$ has an open neighbourhood $W$ with its closure lying on $\mathcal{P}$. Thus the local $\mathcal{P}$ condition reduces to local compactness if $\mathcal{P}$ is the ideal of all compact sets in $X$ and in this case $C_{\mathcal{P}}(X)=C_{K}(X)$

and $C_{\infty}^{\mathcal{P}}(X)=C_{\infty}(X)$. For more information on ideal related problems we refer the articles [3, 4].

The following result is standard and is recorded in [2], Lemma 2.1.

Lemma 6.2. For any finitely many commutative rings $R_{1}, R_{2}, \ldots R_{n}$ each with identity, ideals of the direct product $R_{1} \times R_{2} \times \ldots \times R_{n}$ are precisely of the form: $J_{1} \times J_{2} \times \ldots \times J_{n}$ where for $j=1,2, \ldots n, J_{j}$ is an ideal in $R_{j}$.

We record the following convenient version of the local $\mathcal{P}$ condition for a zerodimensional space $X$.

Theorem 6.3. For a zero-dimensional Hausdorff space $X$, the following statements are equivalent:

(1) $X$ is locally $\mathcal{P}$

(2) $\left\{Z(f): f \in C_{\mathcal{P}}(X) \cap C_{c}(X)\right\}$ is a base for the closed sets in $X$.

(3) $\left\{Z(f): f \in C_{\infty}^{\mathcal{P}}(X) \cap C_{c}(X)\right\}$ is a closed base for $X$.

(4) $\left\{Z(f): f \in C_{\mathcal{P}}(X) \cap C_{c}^{*}(X)\right\}$ is a closed base for $X$.

(5) $\left\{Z(f): f \in C_{\infty}^{\mathcal{P}}(X) \cap C_{c}^{*}(X)\right\}$ is a closed base for $X$.

We omit the proof of this theorem, which can be done by closely following the arguments and making some necessary modifications in the proof of Theorem 4.3 in [2]. We are now ready to enunciate the main theorem in this section.

Theorem 6.4. Let $\mathcal{P} \in \Omega(X)$ where $X$ is a zero-dimensional Hausdorff space which is further locally $\mathcal{P}$. Then the following statements are equivalent:

(1) $C_{\mathcal{P}}(X) \cap C_{c}(X)$ is a Noetherian Ring.

(2) $C_{\mathcal{P}}(X) \cap C_{c}(X)$ is an Artinian Ring.

(3) $C_{\infty}^{\mathcal{P}}(X) \cap C_{c}(X)$ is a Noetherian Ring.

(4) $C_{\infty}^{\mathcal{P}}(X) \cap C_{c}(X)$ is an Artinian Ring.

(5) $X$ is a finite set. 
The proof can be accomplished by making a close introspection of the reasonings made in the proof of the Theorem 1.1 in [2] Nevertheless to make the article selfcontained and to highlight a few important remarks regarding the possible dearth of Noetherian Rings/ Artinian rings lying between $C_{c}^{*}(X)$ and $C_{c}(X)$, we wish to provide an alternatively framed regorous proof of the above theorem.

Proof of the Theorem 6.4 First assume that $X$ is a finite set with ' $n$ ' elements. Then since $X$ is Hausdorff it becomes a discrete space. Consequently $C(X)=\mathbb{R}^{X}$, which is isomorphic to direct product of $\mathbb{R} \times \mathbb{R} \times \ldots \times \mathbb{R}$ ( $n$ times). On the other hand since $X$ is locally $\mathcal{P}$ it follows that $C_{\mathcal{P}}(X)=C_{\infty}^{\mathcal{P}}(X)=C_{c}(X)=C(X)$ consequently $C_{\mathcal{P}}(X) \cap C_{c}(X)=C_{\infty}^{\mathcal{P}}(X) \cap C_{c}(X)=C_{c}(X)=C(X)$. Since the field $\mathbb{R}$ has just 2 ideals, it follows from Lemma 6.2 that $\mathrm{C}(\mathrm{X})$ has just $2^{n}$ many ideals. Hence the rings $C_{\mathcal{P}}(X) \cap C_{c}(X)$ and $C_{\infty}^{\mathcal{P}}(X) \cap C_{c}(X)$ are both Noetherian and Artinian.

Conversely, let $X$ be an infinite set. We shall show that thae $\operatorname{ring} C_{\mathcal{P}}(X) \cap C_{c}(X)$ is not a Noetherian ring. Analogous arguments can be made to show that $C_{\mathcal{P}}(X) \cap$ $C_{c}(X)$ is not an Artinian ring and nor is the ring $C_{\infty}^{\mathcal{P}}(X) \cap C_{c}(X)$ Noetherian or Artinian. As $X$ is an infinite Hausdorff space it contains a copy of $\mathbb{N}(0.13$, [13), So for each $k \in \mathbb{N}$ there exists an open set $W_{k}$ in $X$ such that $W_{k} \cap \mathbb{N}=\{k\}$. Since $X$ is locally $\mathcal{P}$ and zero-dimensional, we can employ Theorem 6.3 to find for each $k \in \mathbb{N}$, an $f_{k} \in C_{\mathcal{P}}(X) \cap C_{c}(X)$ such that $k \in X \backslash Z\left(f_{k}\right) \subset W_{k} \ldots .(1)$. We now assert that the ideal $I=<f_{1}, f_{2}, \ldots f_{k}, . .>$ generated by these $f_{k}^{\prime} s$ in the ring $C_{\mathcal{P}}(X) \cap C_{c}(X)$ can not be finitely generated and hence $C_{\mathcal{P}}(X) \cap C_{c}(X)$ is not Noetherian.(A ring $R$ is Noetherian if and only if each ideal in $R$ is finitely generated: A standard result).

Proof of the assertion: Choose $n \in N$. We show that the ideal $<f_{1}, f_{2}, \ldots f_{n}>\varsubsetneqq I$ and that will do. Indeed from $(0)$ and (1) it follows that $f_{n+1}(n+1) \neq 0$, while $f_{1}(n+1)=f_{2}(n+1)=\ldots=f_{n}(n+1)=0$. Thus there do not exist functions $l_{1}, l_{2}, \ldots l_{n} \in C_{\mathcal{P}}(X) \cap C_{c}(X)$ for which we can write: $f_{n+1}=l_{1} f_{1}+l_{2} f_{2}+\ldots l_{n} f_{n}$. Hence $f_{n+1} \in I \backslash<f_{1}, f_{2}, \ldots f_{k}, . .>$.

Remark 6.5. Since for any $\mathcal{P} \in \Omega(X), C_{\mathcal{P}}(X) \subseteq C_{\infty}^{\mathcal{P}}(X)$ an easy verification, it follows from Theorem 6.3 that for any prescribed ring $R$ lying either between $C_{\mathcal{P}}(X) \cap C_{c}(X)$ and $C_{\infty}^{\mathcal{P}}(X) \cap C_{c}(X)$ or between $C_{\mathcal{P}}(X) \cap C_{c}(X)$ and $C_{\mathcal{P}}(X) \cap C_{c}^{*}(X)$, a zero-dimensional space $X$ is locally $\mathcal{P}$ if and only if $\{Z(f): f \in R\}$ is a base for the closed sets in $X$. With this observation in mind, if we make a close scrutiny into the proof of the converse part of Theorem 6.4, we get the following result.

Theorem 6.6. Given $\mathcal{P} \in \Omega(X)$, if $X$ is an infinite zero-dimensional locally $\mathcal{P}$ space, then no ring lying between $C_{\mathcal{P}}(X) \cap C_{c}(X)$ and $C_{\infty}^{\mathcal{P}}(X) \cap C_{c}(X)$ is Noetherian (respectively Artinian) and also no ring lying between $C_{\mathcal{P}}(X) \cap C_{c}(X)$ and $C_{\mathcal{P}}(X) \cap$ $C_{c}^{*}(X)$ is Noetherian (respectively Artinian).

We record below two special cases of Theorem 6.6, on choosing $\mathcal{P} \equiv$ the ideal of all compact sets in $X$ in the first part of the Theorem and on choosing $\mathcal{P} \equiv$ the ideal of all closed sets in $X$ in the second part of the theorem.

Theorem 6.7. (1) If $X$ is an infinite locally compact zero-dimensional space then no ring lying between $C_{K}(X) \cap C_{c}(X)$ and $C_{\infty}(X) \cap C_{c}^{*}(X)$ is Notherian/ Artinian. 
(2) For any infinite zero-dimensional space $X$, no intermediate ring $A_{c}(X) \in$ $\Sigma_{c}(X)$ is Notherian/Artinian.

\section{Formula FOR $z^{0}$-IDEALS IN INTERMEDIATE RINGS.}

We first show that ideal $I$ in an intermediate ring $A_{c}(X) \in \Sigma(X)$ gives rise to an ideal of closed sets in $X$. Indeed fer any such $I$, we get $\mathcal{P}_{I}^{A_{c}}=\{E \subseteq X: E$ is closed in $X$ and there exists $f \in I$ such that $E \subseteq c l_{X}(X \backslash Z(f))$. If is easy to verify that $\mathcal{P}_{I}^{A_{c}}$ is an ideal of closed sets in $X$ i.e; $\mathcal{P}_{I}^{A_{c}} \in \Omega(X)$ and also that $I \subseteq C_{\mathcal{P}_{I}^{A_{c}}}(X) \cap A_{c}(X) \equiv\left\{f \in A_{c}(X): c l_{X}(X \backslash Z(f)) \in \mathcal{P}_{I}^{A_{c}}\right\}$. The following fact tells decisively when does equality occur in the last inclusion relation. Incidentally we get an explicit formula for $z^{0}$-ideals in the intermediate rings.

Theorem 7.1. Let $A_{c}(X) \in \Sigma_{c}(X)$. Then an ideal $I$ in $A_{c}(X)$ is a $z^{0}$-ideal in this ring if and only if there exists $\mathcal{P} \in \Omega(X)$ such that $I=C_{\mathcal{P}}(X) \cap A_{c}(X)$.

Proof. First assume that $I$ is a $z^{0}$-ideal in $A_{c}(X)$. In view of the observations foregoing this theorem, it is sufficient to show that $C_{\mathcal{P}_{I}^{A_{c}}}(X) \cap A_{c}(X) \subseteq I$. So let $g \in C_{\mathcal{P}_{I}^{A_{c}}}(X) \cap A_{c}(X)$ then $c_{X}(X \backslash Z(g)) \in \mathcal{P}_{I}^{A_{c}}$. Consequently there exists $f \in I$ such that $c l_{X}(X \backslash Z(g)) \subseteq l_{X}(X \backslash Z(f))$. This implies on taking complement in $X$ that $\operatorname{Int}_{X} Z(g) \supseteq \operatorname{Int}_{X} Z(f)$, which further implies in view of Theorem 4.3 that $g \in \mathcal{P}_{f} \equiv$ the intersection of all minimal prime ideals in $A_{c}(X)$ containing $f$. Since $f \in I$ and $I$ is a $z^{0}$-ideal in $A_{c}(X)$ it follows that $g \in I$. Thus we get: $I=C_{\mathcal{P}_{I}^{A_{c}}}(X) \cap A_{c}(X)$.

To prove the other part of the theorem we show that for any $\mathcal{P} \in \Omega(X), C_{\mathcal{P}}(X) \cap$ $A_{c}(X)$ is a $z^{0}$-ideal in $A_{c}(X)$. Choose $f \in C_{\mathcal{P}}(X) \cap A_{c}(X)$, then $c l_{X}(X \backslash Z(f)) \in \mathcal{P}$. We need to verify that $\mathcal{P}_{f} \subseteq C_{\mathcal{P}}(X) \cap A_{c}(X)$. So choose $g \in \mathcal{P}_{f}$, then by Theorem $4.3 \operatorname{Int}_{X} Z(f) \subseteq \operatorname{Int}_{X}(g)$, which implies obviously that $c l_{X}(X \backslash Z(g)) \subseteq c l_{X}(X \backslash$ $Z(f))$. Since $f \in C_{\mathcal{P}}(X)$ it follows that $c l_{X}(X \backslash Z(f)) \in \mathcal{P}$. As $\mathcal{P}$ is an ideal of closed sets in $X$, this further implies that $c l_{X}(X \backslash Z(g)) \in \mathcal{P}$ i.e; $g \in C_{\mathcal{P}}(X) \cap A_{c}(X)$. Thus $\mathcal{P}_{f} \subseteq C_{\mathcal{P}}(X) \cap A_{c}(X)$.

It is established recently in 1], Theorem 5.2 that an ideal $I$ in $C(X)$ with $X$, Tychonoff is a $z^{0}$-ideal in $C(X)$ if and only if there exists $\mathcal{P} \in \Omega(X)$ such that $I=C_{\mathcal{P}}(X)$. Therefore we can make the following comments.

Remark 7.2. $z^{0}$-ideals in the intermediate rings $A_{c}(X) \in \Sigma_{c}(X)$ with $X$, zerodimensional are exactly the contractions of $z^{0}$-ideals in $C(X)$. 


\section{REFERENCES}

[1] Sudip Kumar Acharyya, Sagarmoy Bag, Goutam Bhunia and Pritam Rooj: Some new results on functions in $C(X)$ having their support on ideals of closed sets, Quaest. Math. 42(8) (2019), 1079-1090.

[2] Sudip Kumar Acharyya, Kshitish Chandra Chattopadhyay and Pritam Rooj: A Generalised version of the rings $C_{K}(X)$ and $C_{\infty}(X)$-an enquiry about when they become Noetherian, Appl. Gen. Topol. 16(1), (2015), 81-87.

[3] S.K. Acharyya and S.K. Ghosh: A note on functions in $C(X)$ with support lying on an ideal of closed subsets of X, Topology Proc. 40(2012), 0297-301.

[4] IBID: Functions in $C(X)$ with support lying on a class of subsets of $X$, Topology Proc. 35(2010), 127-148.

[5] F. Azarpanah, O.A.S Karamzadeh, Z. Keshtkar and A.OR.Olfati: On maximal ideals of $C_{c}(X)$ and the uniformity of its localizations, Rocky Mountain J. Math. 48(2) (2018), 345-382.

[6] F. Azarpanah, O.A.S Karamzadeh and R.A. Aliabad: On $z^{0}$-ideals of $C(X)$. Fund. Math. 160(1999), 15-25.

[7] ....: On ideals consisting entirely of zero divisors, Comm. Algebra, 28(2000), 1061-1073.

[8] F. Azarpanah and M.Karavan: On non regular ideals and $z^{0}$-ideals in $C(X)$, Czechoslovak Math. J. 55(2) (2005), 397-407.

[9] Sagarmoy Bag, Sudip Kumar Acharyya and Dhananjoy Mandal: A class of ideals in intermediate rings of continuous functions, Appl. Gen. Topol. no 1 (2019), 109-117.

[10] L.H. Byun and S. Watson: Prime and maximal ideals in subrings of $C(X)$, Topology Appl. 40(1991), 45-62.

[11] P.Bhattacharjee, M.L Knox and W.W. Mcgovern: The classical ring of quotients of $C_{c}(X)$, Appl. Gen. Topol. (2014), 147-154.

[12] R.E Chandler: Hausdorff compactifications, Marcel Dekker, Newyork,1976.

[13] L. Gillman and M. Jerison: Rings of Continuous Functions, Newyork, Von Nostraind Reinhold co, 1960.

[14] M.Ghadermazi, O.A.S Karamzadeh, M.Namdari: On the functionally countable subalgebras of $C(X)$, Rend. Semin. Mat. Univ. Padova Vol 129, (2013), (47-69).

[15] E.Hewitt: E. Hewitt, Rings of real-valued continuous functions I, Trans. Amer. Math. Soc. 64(1948), 54-99.

[16] O.A.S Karamzadeh, Z. Keshtkar: On c-real compact spaces, Quaest. Math. 41(8), (2018), 1135-1167.

[17] J. Kist: Minimal prime ideals in commutative semigroups, Proc. Lond. Math. Soc. 13(1963), 31-50.

[18] R.Levy: Almost P-spaces, Canad. J. Math. 29(1977), 284-288.

[19] J.R Porter and R.G Woods: Extension and absolutes of Hausdorff spaces, springer.verlag, Newyork, 1988.

[20] L.Redlin, S.Watson: Maximal ideals in subalgebras of $C(X)$, Proc. Amer. Math. Soc. 100(4), (1987), 763-766.

[21] : Structure spaces for rings of continuous functions with applications to real compactifications, Fund. Math. 152(1997), 151-163.

[22] J.Sack, S.Watson: Characterizing $C(X)$ among intermediate $C$-rings on $X$, Topology Proc. 45(2015), 301-313.

[23] : $C$ and $C^{*}$ among intermediate rings, Topology Proc. 43(2014), 69-82.

Department of Pure Mathematics, University of Calcutta, 35, Ballygunge Circular ROAD, KOLKATA - 700019, INDIA

E-mail address: sdpacharyya@gmail.com

Department of Pure Mathematics, University of Calcutta, 35, Ballygunge Circular ROAD, KOLKATA - 700019, INDIA

E-mail address: bharti.rakesh292@gmail.com

Department of Pure Mathematics, University of Calcutta, 35, Ballygunge Circular ROAD, KOLKATA - 700019, INDIA

E-mail address: debrayatasi@gmail.com 